\title{
THE ACCURACY OF DETERMINATION OF RADAR SHADOWS
}

\begin{abstract}
Currently, the GIS software is often used to perform various tasks related to spatial planning. Exemplary use of GIS techniques is determination of marine radar shadows. Such analysis can be helpful during creating surveillance systems, which generally consist of several radar stations. But the use of standard analysis tools with the default functionality may not always yield satisfactory results. The reason for this, in the case of radar sensors, is the image distortion caused by the characteristics of the radar beam. This paper presents a modified method for the visibility analysis, which includes two basic radar image distortions - angular and radial distortion. Such improvement gives much more accurate results during determination of radar shadows then application of standard methods of viewshed analysis. Presented method was verified in the aspect of accuracy by using real radar image and radiolocation target. Further accuracy study took into consideration different DSM models used to perform viewshed analyses.
\end{abstract}

\section{Keywords:}

viewshed analysis, radar, navigation, surveillance systems.

\section{INTRODUCTION}

Inland waters are often difficult cases during designing system of observational sensors like radars and CCTV cameras. This situation is caused by irregular shape of river section and occurrence of obstacles, which just limits fields of observations. One of the main targets during designing the net of sensors is to minimize their number and maximize their area of observation. This task requires certain advanced method of spatial planning, which take into account the spatial factors mentioned above. In earlier work for that purpose were used GIS techniques. They allow planning observational sensors by using 3D model of aquatory or by performing raster-based viewshed analyses [8]. It was also noted that standard form of viewshed analysis could not be perfect for radar sensors. The reason was the basic radar distortions depended mainly on the beam characteristic, which can decrease observation area [6]. Thus, the further step was to implement the basic radar distortions for viewshed analysis, which overall improve process of spatial planning of radar sensors. This paper presents the final step of this research, which is verification of proposed 
method in the aspect of radar shadows determination. This scientific work was financed by Polish funds for science in 2010-2012 as a research project.

\section{VIEWSHED ANALYSIS}

Viewshed analysis is a method, which allows determination of visible and invisible areas. In basic form it is conducted by using raster model of Digital Surface Model. Viewshed analysis classifies raster cells into two classes of data. The first class represents visible area from certain point of observation, which is called viewshed [11]. The second class represents area, which is invisible for observer. Information in this form allows for easy evaluation of visible and invisible areas from the sensor location. In current GIS programs can also be performed analysis from different observational point, which helps in designing observational sensor location or the entire sensor network [7, 4]. Such method in a simple way determines the viewsheds. The only problem may be high resolution rasters, which need much more time for data processing.

In previous work also was stated that viewshed analysis should be preceded by visual analysis based on 3D aquatory model [8]. This is due to better perception of visible areas, barrier and platform identification and overall analysis of various spatial factors. Analyses based on 3D aquatory model may also contain smaller objects like masts, cranes, gantries, which are difficult to model by using raster model. Apart from this, analysis can be performed for objects with clearance, like bridges. Analysis using 3D model is much faster and shorten the time needed for determination the initial location of sensors [7].

\section{MODIFIED VIEWSHED ANALYSES}

Basic visibility analysis, performed on 3D vector and raster model, is adequate for optical sensors like CCTV cameras. Unfortunately, radar sensor produce image with distortions. The basic distortions depend on beam characteristic like its horizontal angle and pulse length for pulse radars [1] or electromagnetic wave properties for solid-state radars. More important is beam horizontal angle, because it increases in proportion to the distance. Even small values of beam angle can produce large distortion for greater distances. During planning radar sensors for inland water this factor play the main role, because width of river section can be relatively small. For analyzed areas river sections have width from 100 to $400 \mathrm{~m}$. For radar sensor network in port of Szczecin are considered radars with small antennas due to installation reasons and their low power, what is important in urbanized areas. But this devices 
produce beam up to $5.2^{\circ}$, what dramatically enlarge distortion. Without consideration of such distortion application of standard viewshed analysis can lead to not quite accurate results and wrong assessment of radar shadows.

In this research was used modified viewshed analysis. It was created by adding radar distortions for standard method of viewshed analysis Distortions were calculated by entering three parameters: beam width in degrees, radial distortion in meters and sensor position. Results of analyses were presented in georeferenced raster format. For this purpose was written dedicated computer program. The first studies with this method were presented in previous work [3]. Comparison of standard viewshed analysis with modified viewshed analysis for beam of $1.2^{\circ}$, where radial distortion equals $8.25 \mathrm{~m}$ are illustrated in fig. 1 .

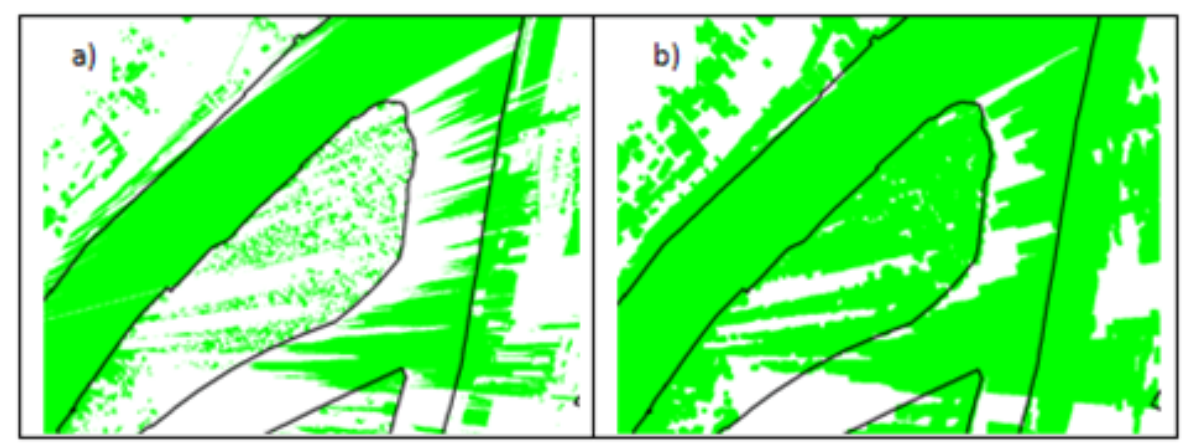

Fig. 1. Results of viewshed analysis: a) standard method; b) modified method [own study]

\section{METHOD VERIFICATION}

The developed method was verified using the real radar image of the pulse radar situated on the roof of Maritime University. Radar was working in the mode of short pulse $(55 \mathrm{~nm})$.Verification was conducted for sections of the river fairway behind the Grodzka Island. To create radar shadow areas, i.e. invisible areas for radar sensor, was used as radiolocation target the school vessel 'Hydrograf XXI'. It sailed through the planned route behind the Grodzka Island (fig. 2).

In the studies was used a module of Maris radar with software ARPA 2000, which was connected to the transceiver circuit and worked in slave mode. Registration lasted about 3.5 hours; the entire image was recorded in video format. Before images registration the radar was calibrated by a technician and verified by measuring distances to test land targets. The set of images used for further research was collected by saving screenshots. View of radar station in the laboratory and single screenshot with radar image is illustrated in figure 3. 

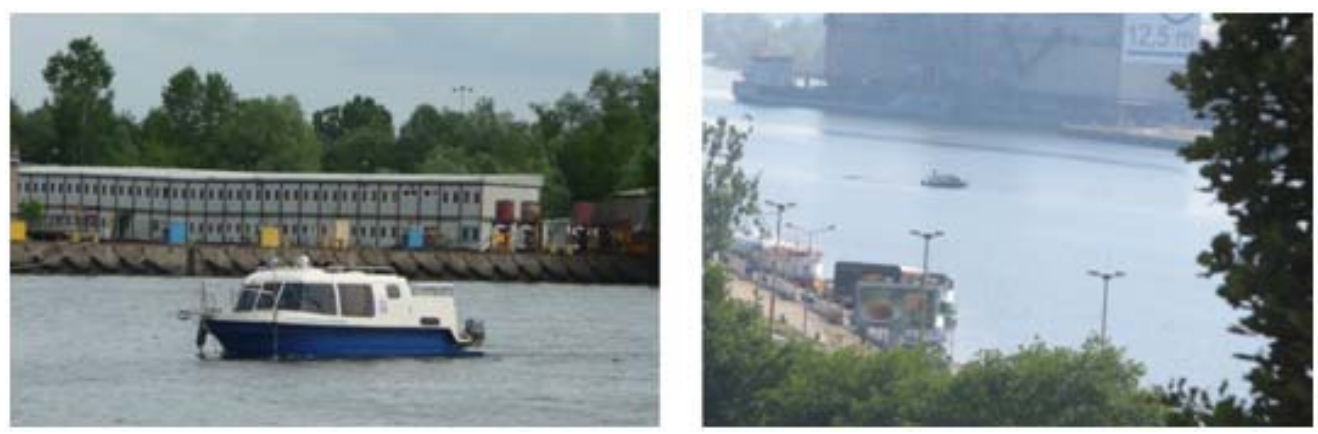

Fig. 2. School vessel used in the role of radiolocation target [own photos]
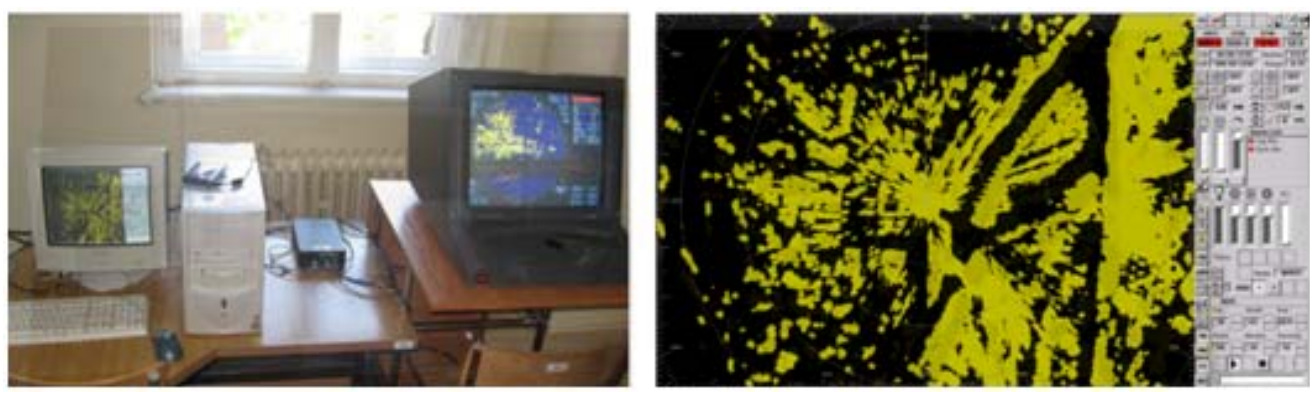

Fig. 3. Radar station in the laboratory and example of recorded radar image [own study]

In the next step the mosaic was created from 1770 recorded radar images (fig. 4). By conducting this operation was obtained the combined image, which aggregated echoes originating from the school vessel. Combined echoes were used later to determine the actual radar shadow areas and also areas under continuous observation. Having final radar image, it was registered to the Polish national coordinate system PUWG 2000, which allowed conducting the appropriate analyses in the project by using ArcGIS 10.0 software. Error of image georeferencing was calculated by using 9 land points measured by GPS/RTK set and equaled to $2.92 \mathrm{~m}$.

After the mosaicsing a radar image was synthetic enhanced by aggregated random noises or distortion caused by radar system. Therefore, radar image was filtered of the isolated echoes of the area below $1000 \mathrm{~m}^{2}$ and the echoes situated behind of the tall trees of Grodzka Inland. These kinds of echoes do not constitute significant information in the sense of continuity of observation or tracking. Part of radar image used in the studies before and after filtering is illustrated in figure 5. 


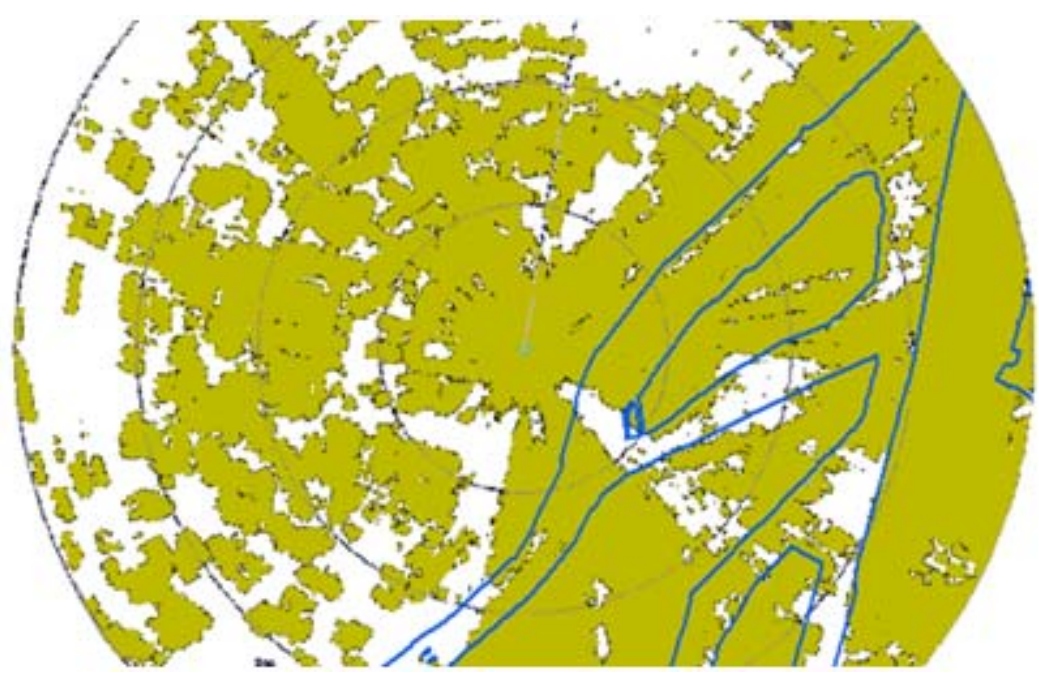

Fig. 4. Mosaics of registered single radar images [own study]
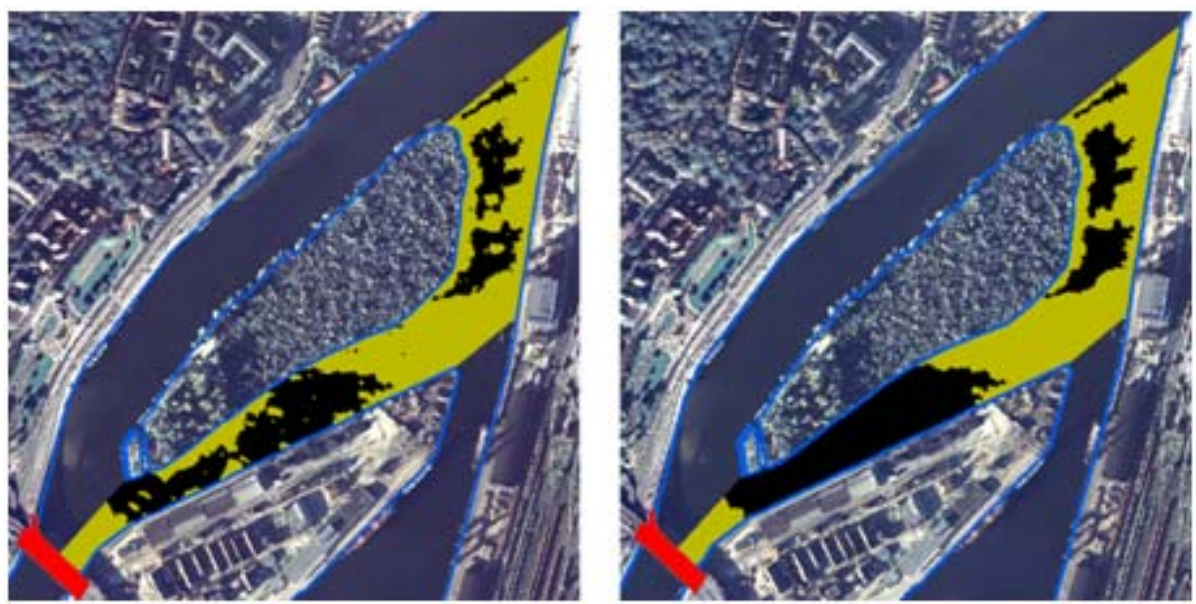

Fig. 5. Studied part of radar image behind Grodzka Island: on the left - original radar image, on the right — filtered radar image [own study]

\section{ANALYSIS AND RESULTS}

Analysis was conducted on various form of DSM of Grodzka Island, which area in the more part was afforested. Such geographical objects are very difficult to model due to their irregular shapes created by tree crows (fig. 6). The reason of various DSM applications was to check their influence on final results. By comparing these results is possible to asses each DSM in the aspect of accuracy of radar shadows generation. 


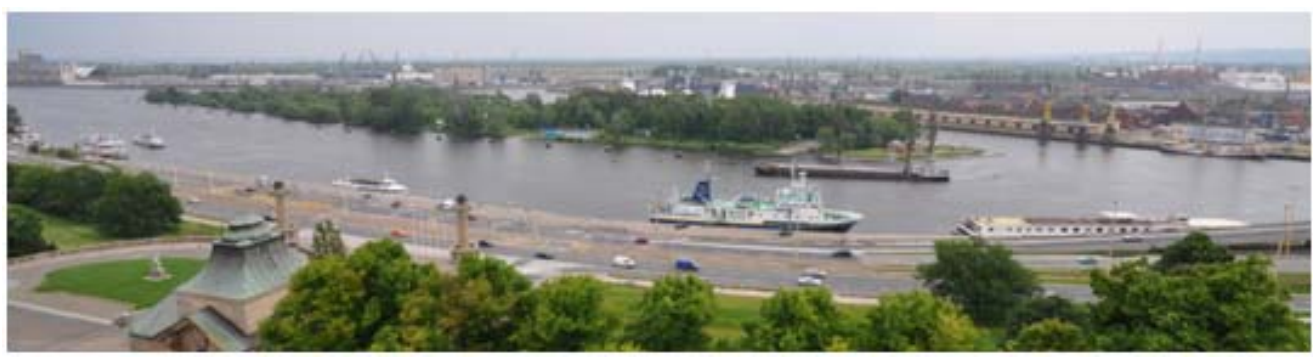

Fig. 6. View on Grodzka Island from radar sensor position [own photo]

Thus, for research purpose, was created DSM by following surface modeling method: kriging with linear variogram model, spline, natural neighbor (fig. 7) and TIN (fig. 8). All algorithms were implemented in ArcGIS 10.0 software with Spatial Analyst extension. Each method has specific properties, which were detailed described in previous publication [12]. Additionally, all this methods were compared with model LOD1 (fig. 8). This model was created by extrusion vegetation polygons to average height of trees according to the CityGML standard [10]. Polygons of trees with dominant heights were created by manual classification. Heights of trees for elaboration of above method were measured by GPS/RTK set with TruPulse 360 B distance meter. Finally all above methods were compared with the model prepared from LIDAR data acquired from airborne laser scanning (fig. 8). This kind of data is the most accurate due to its density, vertical and horizontal accuracy. High data density enables to prepare very accurate surface model, especially in the case of features with irregular shapes. For elaboration of DSM model from LIDAR data were used data from first return of points cloud [2], where each cell of raster model represents their maximum value.

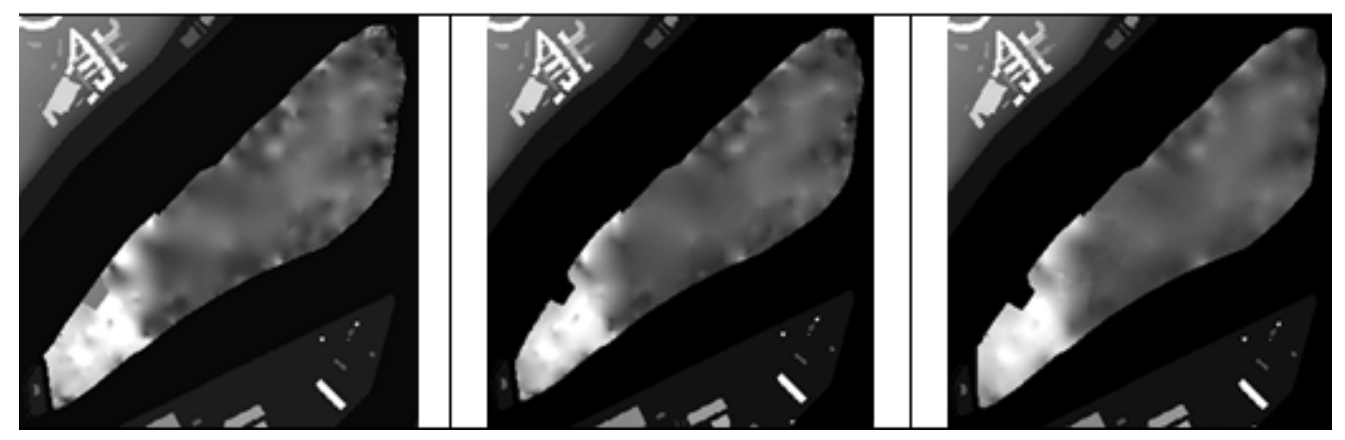

Fig. 7. DSM of Grodzka model elaborated by using spline, natural neighbor and kriging [own study] 


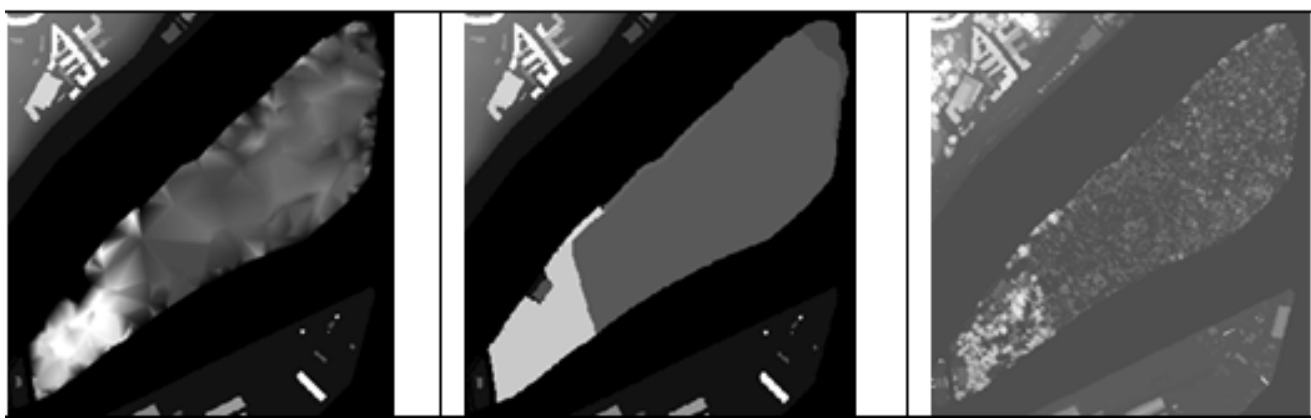

Fig. 8. DSM of Grodzka model elaborated by using TIN, LOD1 and LIDAR data [own study]

In the case of dedicated radar sensor (NUCLEUS), the following parameters were used to generate a distortion of radar: a radar beam horizontal angle of $1.2^{\circ}$, radial distortion of $8.25 \mathrm{~m}$ for short pulse length of $55 \mathrm{~nm}$, vertical beam angle of $20^{\circ}$. Range of analyzed waterways covered the area behind the Grodzka Island. Result rasters of modified viewshed analysis for DSM elaborated by using spline, natural neighbor and kriging are illustrated in fig. 9 and for TIN, LOD1 and LIDAR data in fig. 10.

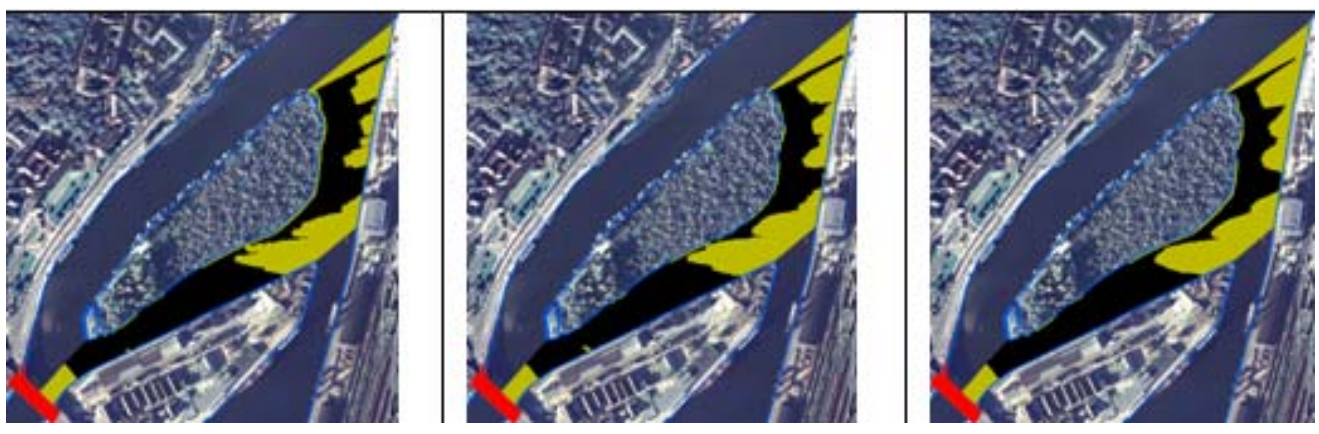

Fig. 9. Results of modified viewshed analysis for spline, natural neighbor and kriging DSM [own study]

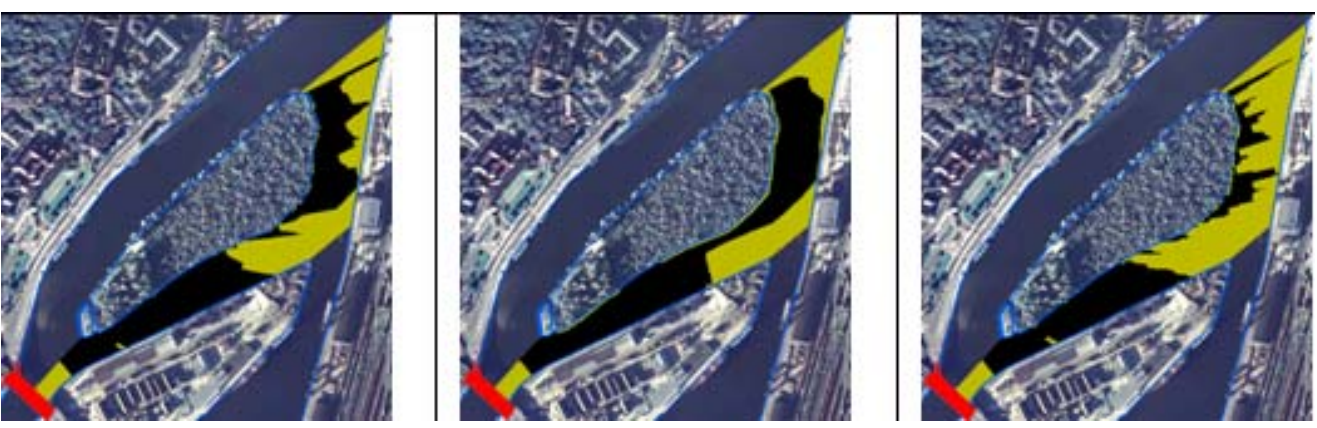

Fig. 10. Results of modified viewshed analysis for TIN, LOD1 and LIDAR DSM [own study] 
The effectiveness of the modified method of visibility analysis was estimated by comparing the invisible areas by the proposed ratio of compliance of radar shadow determination. This factor takes into account different models of DSM in terms of determining the radar shadow of the analyzed area. It was calculated using the following own formula:

$$
R_{i}=100 *\left(1-\left|\frac{A_{i}-A_{r}}{A_{o}}\right|\right)[\%]
$$

where:

$A_{i}$ - radar shadow area for a particular model of DMS,

$\mathrm{i}=1 . .6$,

where:

1 - LIDAR, 2 - kriging, 3 - natural neighbor, 4 - TIN, 5 - spline, 6 - LOD1;

$A_{r}$ - area radar shadow for real radar image;

$A_{o}$ - the total area covered by the study.

Another comparative criterion was visual assessment of the shape of the resulting radar shadow area. The second criterion is related to the use of different DSMs for the Grodzka Island, which further implies the need to assess invisible areas convergence in relation to the real radar shadows.

First results show that sizes of visible and invisible areas are different. Generally, most methods tend to increase invisible areas and shrink visible ones (fig. 11). The most convergent results were obtained for the model built from LIDAR data, but invisible areas are a bit smaller than the real ones. Next in the order was method of kriging, natural neighbor, TIN and spline. The smallest convergence to the real radar shadows was obtained for LOD1 model. Figure 11 presents also area of synthetic distortions, which are significant part of the whole area. Without them the differences between visible and invisible areas would be much bigger.

Figure 12 shows values of ratio of compliance of radar shadow determination. DSM model created from LIDAR data was the best during application of modified viewshed visibility $\left(R_{1}=99.6 \%\right)$. DSM elaborated by kriging and natural neighbor produced results of $R_{2}=87.7 \%$ and $R_{3}=86.9 \%$. For TIN, spline, LOD1 were obtained following values: $R_{4}=84.7 \%, R_{5}=82.8 \%, R_{6}=80.8 \%$. 


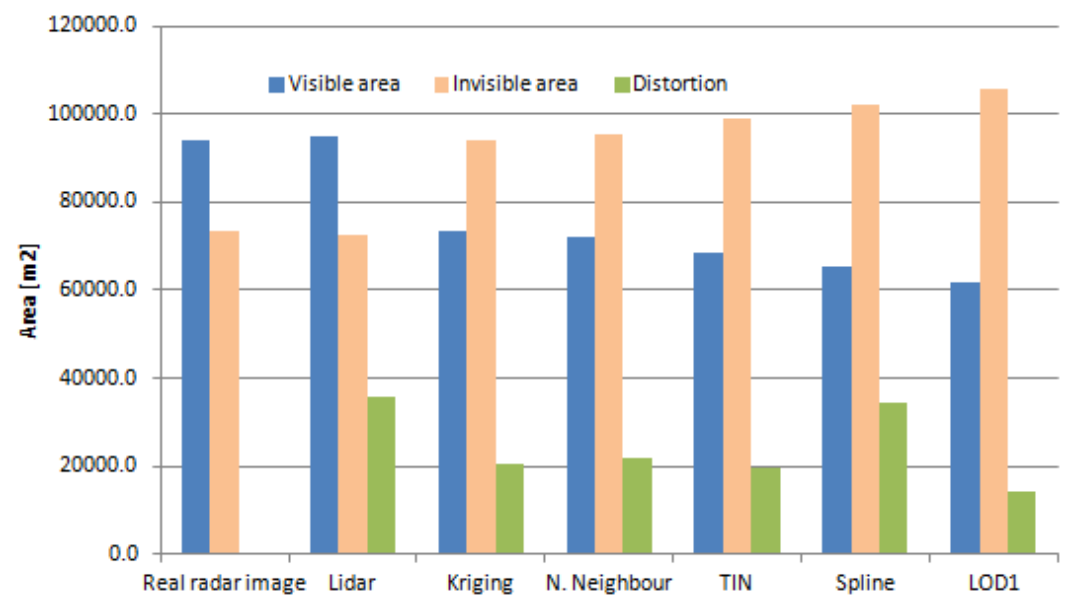

Fig. 11. Comparison of visible and invisible areas for studied DSMs [own study]

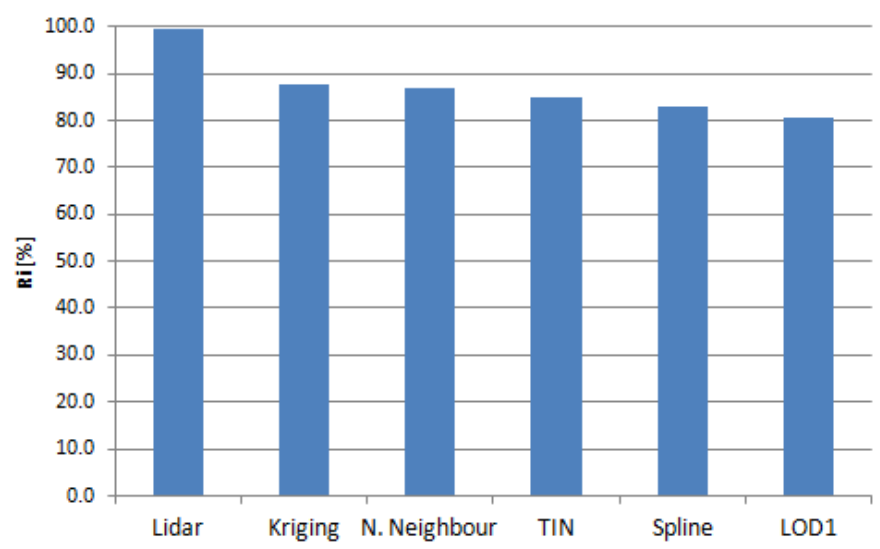

Fig. 12. Values of ratio of compliance of radar shadow determination [own study]

The visual assessment of areas of visible and invisible pointed to convergence with the areas of the real radar image. Comparing images from fig. 9 and fig. 10 with the real radar image (fig. 5) may be stated that general outlines of considering areas are correct. More similar areas were created by more advanced method of surface modeling like kriging, natural neighbor, TIN and spline and LIDAR data. A characteristic feature of these models is the possible mapping of smaller areas, which is associated with a more accurate shape of the modeled surface. LOD1 model, which has the biggest simplification of the real surface, presented studied areas more generalized. On the one hand, comparing with the numerical results, it is the worst value. But on the other hand, this generalization shows the areas that are relevant to the need to maintain the continuity of visual target observation, including the tracking. 


\section{ASPECTS OF ANALYSES UNCERTAINTY}

Uncertainty of analysis is directly related to the accuracy of measurements and further stages of data processing. It can be expressed like the lack of knowledge of final exact accuracy. An example of data processing is the creation of DSM in the form of continuous surfaces by various interpolation methods. Another aspect is the raster resolution, which because of its structure in some way affects the generalization of the modeled phenomenon. Another reason of analyses uncertainty is the software. Depending on the manufacturer during programs creations are used different compilers, algorithms, which also can affect the final result.

In the case of different methods used in this research for creation of numerical model of tree crowns, we can conclude that these differences are quite significant. In comparison with the model created from LIDAR data the results obtained by using other methods were worse for $10-20 \%$. It is hard to directly determine the accuracy of the created model. Often used in the calculation the error based on an independent test dataset is not always reliable, due to the lack of choice of the optimal testing subset. The identification of the optimal testing data subset should result in separation of the next data subset for its validation, which in turn leads to an open optimization criterion. During creation of a statistically continuous surface a good approach is application of the method, which modeling properties for reconstruction of various phenomena have been confirmed on the basis of researches and experiments. In this paper, one of these methods is kriging, which is another example of the confirmation of its universality.

Another factor influencing the uncertainty analysis is the resolution of the raster model. Generally, the resolution should be as high as possible, but due to the scope of the elaboration can be determined in terms of efficiency of implemented algorithms or computational capabilities of the computer. In this work for generation of modified viewshed analysis was used resolution of $1 \mathrm{~m}$, while for standard method was used $0.25 \mathrm{~m}$ due to more efficient algorithm in ArcGIS software. The impact on the visibility analysis results are shown on the example of DSM obtained from LIDAR data, because it was the most accurate. As can be seen in table 1, together with the reduction of raster resolution were increased the visible areas and automatically decreased invisible. Reference raster has resolution of $0.25 \mathrm{~m}$. This is due to averaging the elevation values in larger cells of the raster, which causes a gradual smoothing. In the percentage measure in relation to the reference raster, the percentage loss of the invisible areas increases by about $10 \%$ for a resolution of $2 \mathrm{~m}$. 
Table. 1. Percentage loss of the invisible areas for various raster resolutions

\begin{tabular}{|l|c|c|c|c|c|}
\hline Raster resolution [m] & 0.5 & 1 & 2 & 5 & 10 \\
\hline Percentage loss of invisible areas [\%] & 3 & 7 & 10 & 18 & 26 \\
\hline
\end{tabular}

Another issue is compatibility of analysis depends on the software types. On the basis of previous comparisons of visibility analyzes in ArcGIS and ERDAS Imagine software [7] these differences were relatively small $\left(1953 \mathrm{~m}^{2}\right)$ compared with the influence of raster resolution.

\section{SUMMARY}

In this paper was presented modified method of viewshed analysis and its verification in the aspects of radar shadows determination. The basic modification was related to basic radar image distortions, which were added to new method. Studies showed that synthetic distortions significantly improved results of analyses. An important factor is the type of DSM. The best results were obtained by using DSM model from LIDAR data. Invisible area in this case was a bit lower compared with real radar shadows. This happened probably due overall tendency to lowering heights of trees by application of this technology [9]. For this reason, LIDAR data should be acquired during the full vegetation period, what increases accuracy of DSM creation. In some special cases, where the tree crowns are loose, predicted invisible areas can be much smaller. From other methods, the best results were obtained for DSM created by kriging. Last method was LOD1 model, which on the other hand, showed more distinctly areas of constant observation. Also an important role plays raster resolution. By increasing raster cells, accuracy of analyses decreases by enlargement of visible areas. Taking all above factors into consideration during radar sensor planning and estimating radar shadows areas should brings much better results than applying standard viewshed analyses.

\section{REFERENCES}

[1] Bole A. G., Dineley W. O., Radar and ARPA Manual, Butterworth Heinemann, Reissue edition, 1993.

[2] GISPRO, Photogrammetry \& Geosciences company, Source of LIDAR Points Cloud, Szczecin 2011. 
[3] Łubczonek J., Kazimierski W., Pałczyński M., Planning of combined system of radars and CCTV cameras for inland waterways surveillance by using various methods of viewshed analysis, Proceedings of International Radar Symposium IRS, Lipsk 2011.

[4] Lubczonek J., Stateczny A., Aspects of spatial planning of radar sensor network for inland waterways surveillance, Proceedings of the $6^{\text {th }}$ European Radar Conference, Rome 2009.

[5] Łubczonek J., Stateczny A., Spatial planning of land-based marine radar sensors by using 3D carthographical model of the port and urbanized areas, Proceedings of CoastGIS 2011, Oostend 2011.

[6] Łubczonek J., Application of GIS technique in VTS radar stations planning. Proceedings of International Radar Symposium IRS 2008, A. Kawalec, P. Kaniewski (ed.), Wrocław 2008 (CD edition).

[7] Łubczonek J., Evaluation of functionality of ArcGIS and Erdas Imagine programms in the aspect of the spatial planning of the observational sensors, Annals of Geomatics, 2011, Vol. IX, No. 2.

[8] Łubczonek J., Location of radar stations based on viewshed analysis, Polish Journal of Environmental Studies, 2007, Vol. 16, No. 6B.

[9] Marmol U., Będkowski K., Accuracy of tree height estimation using lidar dataderived DCM, Archives of Photogrammetry, Cartography and Remote Sensing, 2008, Vol. 18.

[10] Open Geospatial Consortium Inc., OpenGIS City Geography Markup Language (CityGML) Encoding Standard ver. 1.0.0, http://www.citygml.org/,2008.

[11] Smith M., Goodchild M., Longley P., Geospatial Analysis - a comprehensive guide to Principles, Techniques and Software Tools. $3^{\text {rd }}$ edition, 2009.

[12] Stateczny A. (ed.), Comparative Navigation (in Polish), Gdańsk Science Society, Gdańsk 2006.

Received May 2012

Reviewed November 2012 